\title{
Approximate spacetime symmetries and conservation laws
}

\author{
Abraham I. Harte \\ Enrico Fermi Institute \\ University of Chicago, Chicago, IL, 60637, USA \\ E-mail: harte@uchicago.edu
}

\begin{abstract}
A notion of geometric symmetry is introduced that generalizes the classical concepts of Killing fields and other affine collineations. There is a sense in which flows under these new vector fields minimize deformations of the connection near a specified observer. Any exact affine collineations that may exist are special cases. The remaining vector fields can all be interpreted as analogs of Poincaré and other well-known symmetries near timelike worldlines. Approximate conservation laws generated by these objects are discussed for both geodesics and extended matter distributions. One example is a generalized Komar integral that may be taken to define the linear and angular momenta of a spacetime volume as seen by a particular observer. This is evaluated explicitly for a gravitational plane wave spacetime.
\end{abstract}

PACS numbers: 02.40.-k, 04.20.Cv, 04.25.-g

\section{Introduction}

It is rarely possible to model realistic physical systems with exact solutions to the equations of some general underlying theory. Despite this, many interesting problems deviate only slightly from a model problem that can be understood exactly. Such solutions are usually tractable only because of symmetry assumptions. Once they're understood, perturbation theory may be used to understand many systems that "almost" satisfy the appropriate symmetry principle. While this statement has a clear intuitive meaning, quantifying it can be difficult. It is also not necessarily obvious how - or if it is meaningful - to uniquely propagate a symmetry from solutions where it is exact into the perturbations that break it. These issues are particularly important in the context of conservation laws. As an example, one might want to know how to construct approximately conserved quantities that are unique generalizations of some exact counterpart in a similar system. There would hopefully be a sense in which such quantities varied slowly for some class of small perturbations.

Some steps towards understanding problems like these are explored here in the context of affine collineations (of which Killing vectors are special cases) associated with curved spacetimes. While these kinds of symmetries rarely exist, there are various senses in which approximate replacements can usually be introduced. One method for finding vector fields that are "almost Killing" is to write down an action whose value provides some sense for how nearly a particular flow preserves the metric [1]. There are important caveats to this interpretation, although the final result is that any vector 
field extremizing such an action satisfies a fairly simple generalization of Killing's equation. Various reasons have been given for suggesting other extensions as well [2, 3, 4, 5, 6, While any genuine Killing vectors that might exist are solutions to all of these equations, it is not usually clear how the remaining fields should be interpreted. This problem arises even in flat spacetime.

What form an approximate symmetry should take is highly dependent on its intended use. One application is in the estimation of a black hole's angular momentum. This requires finding rotational Killing fields on certain 2-spheres foliating a horizon. Various methods have therefore been developed for defining such objects using only the intrinsic geometry of these surfaces [7, 8, 9]. The concept of approximate Killing fields has also been adapted for use on initial data sets used in $3+1$ splits of Einstein's equation [10].

The approach taken here is to define a set of vector fields in a four dimensional volume that can all be viewed as analogs of known symmetries in Minkowski spacetime. The physical interpretation is that these fields may be viewed as generators of approximate symmetries by a specified observer. Any sufficiently small region near a particular point can be made to look nearly flat. Some structures from Minkowski spacetime may be therefore be introduced very near this point. Approximate symmetries that take advantage of this fact are proposed in Sect. 2. It is then shown in Sect. 3 that analogous objects can also be introduced near an observer's worldline. These sorts of vector fields can actually be extended in a non-perturbative way to finite regions around the point or worldline from which they were constructed. A well-defined subset provides a precise analog of the Poincaré group. Translations, rotations, and boosts very near an observer extend in a useful way to cover large portions of the spacetime. Any exact symmetries that may exist are included as special cases. Some connections to conservation laws are discussed in Sect. 4. and a simple example involving gravitational plane wave is finally presented in Sect. 5

\section{Exact symmetries}

Given some spacetime $\left(\mathcal{M}, g_{a b}\right)$, there are several types of exact symmetries that may be discussed. The most common of these take the form of vector fields whose associated diffeomorphisms preserve some geometric structure. The most ubiquitous examples are the Killing fields. Their flows preserve the metric. Vector fields $Y_{\mathrm{K}}^{a}$ with this property satisfy

$$
\mathcal{L}_{Y_{\mathrm{K}}} g_{a b}=0 .
$$

Any solutions that may exist can be used to find conserved quantities associated with geodesics or matter distributions [11, identify mass centers [12, simplify Einstein's equation [13, 14, classify its solutions [14, 15, and so on.

This utility has (among other reasons) motivated various generalizations of (1). Perhaps the simplest of these arises from considering flows that preserve the metric only up to some constant factor:

$$
\mathcal{L}_{Y_{\mathrm{H}}} g_{a b}=2 c g_{a b} .
$$

Any $Y_{\mathrm{H}}^{a}$ satisfying this equation with constant $c$ is known as a homothetic vector field or homothety. Allowing the dilation factor $c$ to vary would define a conformal Killing vector. These objects preserve the metric up to an arbitrary multiplicative factor. The standard Killing vectors are special cases of either class. Like them, conformal and homothetic vector fields usually do not exist. Their presence can be very useful, 
however. The existence of a proper (non-Killing) homothetic vector is often used to define a notion of geometric self-similarity, for example. Such objects therefore appear in certain models of gravitational collapse and cosmology. They are also related to the appearance of critical phenomena in general relativity [16].

A simple generalization of the homotheties can be found by considering vector fields that satisfy

$$
\nabla_{a} \mathcal{L}_{Y_{\mathrm{A}}} g_{b c}=0 .
$$

Solutions to this equation are known as affine collineations. They are the generators of infinitesimal affine transformations. Killing and homothetic vector fields are special cases. All of the affine collineations may be interpreted geometrically as preserving the Levi-Civita connection. This means that $\mathcal{L}_{Y_{\mathrm{A}}}$ and $\nabla_{a}$ commute when acting on arbitrary tensor fields. Geodesics and their affine parameters are also preserved under the action of any $Y_{\mathrm{A}}^{a}$. Although this might seem to be a significant generalization of the Killing vector concept, solutions rarely exist. The only non-flat vacuum spacetimes that admit non-homothetic affine collineations are the $p p$-waves [17. Similarly, it has been shown that proper homotheties cannot exist in any asymptotically flat vacuum spacetime with positive Bondi mass [18. Despite these results, interesting affine collineations can occasionally be identified in geometries that are not Ricci-flat. Doing so provides a number of simplifications for various problems. Some of these derive from the fact that

$$
K_{a b}=\mathcal{L}_{Y_{\mathrm{A}}} g_{a b}
$$

is a second-rank Killing tensor; i.e.

$$
\nabla_{(a} K_{b c)}=0 .
$$

It should be noted that not all symmetric tensors satisfying this equation can be derived from affine collineations. One counterexample is the Killing tensor associated with Carter constants in Kerr.

Transformations generated by affine collineations can be viewed as mapping geodesics into geodesics. They preserve the affine parameters of each curve. Dropping this latter requirement recovers the so-called projective collineations. A precise definition may be found in [15, although it will not be needed here. One of their interesting consequences is that they leave invariant the projective curvature tensor:

$$
\mathcal{L}_{Y_{\mathrm{P}}}\left(R_{b c d}^{a}-\frac{2}{3} \delta_{[c}^{a} R_{d] b}\right)=0 .
$$

Vector fields satisfying this equation are not always projective, however.

The list of definitions here could keep growing as new fields are added that preserve more and more geometric structures. Interestingly, the quantities introduced so far all share a very useful characteristic that does not easily generalize: the space of vector fields in each of the mentioned classes has finite dimension. Furthermore, any single element is uniquely determined by its value and the values of its first one or two derivatives at a single point. These properties are well-known for Killing fields. Four dimensional spacetimes (which is all that will be considered here) admit a maximum of 10 linearly independent Killing vectors. At most one homothety can exist that is not itself Killing. The maximum number of (not necessarily proper) conformal Killing vectors is 15 , and the affine collineations total no more than 20 . Finally, the space of projective collineations has a maximum of 24 dimensions [15. Properties like these do not hold for vector fields whose flows leave invariant the Riemann, Ricci, or 
Einstein curvature tensors of a given spacetime. Despite this, the class of approximate symmetries introduced below is constructed so as to have finite dimension. Any given member is fixed by its value together with the values of its first derivatives at a point. Unlike the exact symmetries, these objects always exist at least in some finite region. After fixing a reference frame, the space of approximate symmetries has exactly 20 dimensions. Ten of these will be identifiable as generalized Killing vectors, while the remaining ten will be related to more general affine collineations.

It has already been remarked that the presence of Killing fields implies the existence of various conserved quantities. The same can also be said for more general collineations. Extensive discussions of exact symmetries and associated integrals of the geodesic equation may be found in [19]. Many of these symmetries are non-Noetherian in the sense that they preserve the equations of motion, but not the action. Despite this, their presence allows constants of motion to also be assigned to arbitrary stressenergy distributions satisfying Einstein's equation [20, 21. As will be discussed in Sect. 4 generalizations of these quantities can be associated with any approximate affine collineations that are identified.

\section{Symmetries near a point}

Generic symmetries in general relativity are usually discussed in the context of asymptotically flat spacetimes. There then exist approximate notions of isometry that improve as one approaches infinity [11. Generalizations of these ideas also exist for geometries with somewhat more complicated (but still highly symmetric) asymptotic behavior like that of anti-de Sitter [22]. The assumption of a simple limiting form for the geometry makes it convenient to invariantly describe certain properties of a spacetime in terms of "measurements at infinity." Quantities that may be identified as a spacetime's total energy or angular momentum appear naturally, for example.

While useful in many contexts, these ideas do not always translate into observations made by physical observers. Measurements like those expected from gravitational wave detectors do come very close to fitting into this formalism. Others can require a more local description. In particular, it is sometimes important to understand what given observers would experience inside strongly curved regions of spacetime. Abstracting the concept of an observer to a timelike worldline $\Gamma$, vector fields may be introduced in (say) some convex neighborhood $W$ of $\Gamma$ that act like approximations to Killing fields or more general collineations. This is always possible, and the symmetries these vectors generalize become exact on $\Gamma$ itself. Limiting collineations can evidently be useful on scales that are either very large or very small. It is much less clear how to easily describe systems at intermediate distances.

\subsection{Motivation}

The idea of a local symmetry just outlined is best introduced by first considering vector fields $\psi^{a}(x, \gamma)$ that generalize the affine collineations in some reasonable way near a fixed reference point $\gamma$. Let these objects be defined inside a normal neighborhood $N$ of this point. It is intuitively obvious that vector fields may always be chosen such that $\nabla_{a} \mathcal{L}_{\psi} g_{b c}$ vanishes at $\gamma$. While this condition is reasonable to require, it is not very interesting by itself. Much more can be said if each vector field in this class is uniquely fixed in $N$ by knowledge of

$$
\psi^{\mathrm{a}}(\gamma, \gamma), \quad \nabla_{\mathrm{a}} \psi^{\mathrm{b}}(\gamma, \gamma) .
$$


It will be assumed that each $\psi^{a}$ depends linearly on this initial data with no degeneracy. This implies that there are always $4+16=20$ linearly independent vector fields defined about any given point in a four dimensional spacetime. Note that indices in (7) have been written in a sans-serif font to emphasize that they are associated with the preferred point $\gamma$.

Approximate affine collineations with the appropriate properties may be constructed by projecting symmetries of the tangent space $T_{\gamma} N$ into $N$ using the exponential map. Consider the linear transformations

$$
X^{\mathrm{a}} \rightarrow X^{\mathrm{a}}+\epsilon B_{\mathrm{b}}{ }^{a} X^{\mathrm{b}}
$$

of vectors $X^{\mathrm{a}}$ in this space parameterized by an arbitrary tensor $B_{\mathrm{b}}{ }^{\mathrm{a}}$. Being a vector space, $T_{\gamma} N$ has a preferred origin. Adding another term to (8) to shift that origin would be awkward. The translational symmetries that such a procedure might produce are certainly important, although generating them requires a more subtle treatment described below. For now, consider only the vector fields

$$
\Psi^{\mathrm{a}}=X^{\mathrm{b}} B_{\mathrm{b}}{ }^{\mathrm{a}}
$$

associated with homogeneous transformations of the given form. These clearly satisfy

$$
\frac{\partial}{\partial X^{\mathrm{a}}} \mathcal{L}_{\Psi} g_{\mathrm{bc}}=2 \frac{\partial}{\partial X^{\mathrm{a}}}\left(g_{\mathrm{d}(\mathrm{c}}(\gamma) \frac{\partial}{\partial X^{\mathrm{b})}} \Psi^{\mathrm{d}}\right)=0,
$$

so they are affine within $T_{\gamma} N$ in the sense of (3). Such transformations can be made to induce shifts $x \rightarrow x+\epsilon \psi$ in spacetime points associated with vectors $X^{\text {a }}$ via

$$
x=\exp _{\gamma} X .
$$

A simple relation between $\psi^{a}$ and $\Psi^{a}$ is found by introducing Synge's world function $\sigma(x, y)=\sigma(y, x)$. This two-point scalar returns one-half of the squared geodesic distance between its arguments. The assumption that $N$ be a normal neighborhood of $\gamma$ ensures that $\sigma(x, \gamma)$ is uniquely defined for all points $x$ in this region. Many of its properties are reviewed in [23, 24]. Most importantly for the problem at hand, the first derivative of the world function effectively inverts the exponential map. Any set $\left\{\gamma, x, X^{a}\right\}$ satisfying (11) is related via

$$
X_{\mathrm{a}}=-\sigma_{\mathrm{a}}(x, \gamma) \text {, }
$$

where the common shorthand $\sigma_{\mathrm{a}}=\nabla_{\mathrm{a}} \sigma=\partial \sigma / \partial \gamma^{\mathrm{a}}$ has been used. The right-hand side of (12) generalizes the concept of a separation vector between two points. It is useful in that a straightforward expansion shows that linear transformations of the form (8) effectively shift spacetime points by an amount parameterized with a vector $\psi^{a}(x, \gamma)$ satisfying

$$
\Psi^{\mathrm{a}}=-\sigma^{\mathrm{a}}{ }_{a} \psi^{a} .
$$

If the various components of $X^{\mathrm{a}}$ as defined in (12) are used as coordinates, the bitensor $-\sigma^{\mathrm{a}}{ }_{a}=-g^{\mathrm{ab}} \partial^{2} \sigma / \partial x^{a} \partial \gamma^{\mathrm{b}}$ reduces to the identity. Components of $\psi^{a}$ and $\Psi^{\mathrm{a}}$ are therefore identical in normal coordinate systems of this type. In general, it is useful to introduce

$$
H^{a}{ }_{a}=\left[-\sigma_{a}^{a}\right]^{-1}
$$

as the matrix inverse of the operator appearing in (13). This always exists in the regions considered here. Using (9) now shows that

$$
\psi^{a}(x, \gamma)=-H^{a}{ }_{\mathrm{a}} \sigma_{\mathrm{b}} B^{\mathrm{ba}} .
$$


Holding $\gamma$ fixed, this equation defines a 16-parameter family of vector fields generated by $B_{\mathrm{ab}}=\nabla_{\mathrm{a}} \psi_{\mathrm{b}}(\gamma, \gamma)$. Every such $\psi^{a}$ vanishes at $\gamma$. It also satisfies (3) at this point. In flat spacetime, these vector fields coincide everywhere with exact affine collineations.

Not all such symmetries are included in (15), however. The four translational Killing fields are missing. These can be obtained by considering transformations that directly shift the base point $\gamma$. Perturbations of this form cannot leave $X^{\text {a }}$ fixed, as the initial and final vectors must be elements of different spaces. Introducing some $A^{\text {a }}$, we therefore demand that $X^{\text {a }}$ be parallel-transported along the curve that $\gamma$ follows under the one-parameter family of transformations

$$
\gamma \rightarrow \gamma+\epsilon A \text {. }
$$

Using this together with (12) and the homogeneous transformation (8) generates the full 20-parameter family of approximate affine collineations

$$
\psi^{a}=H^{a}{ }_{\mathrm{a}}\left(\sigma^{\mathrm{a}}{ }_{\mathrm{b}} A^{\mathrm{b}}-\sigma_{\mathrm{b}} B^{\mathrm{ba}}\right) .
$$

Given any $A^{\mathrm{a}}$ and $B^{\mathrm{ab}}$, these objects all satisfy

$$
\nabla_{\mathrm{a}} \mathcal{L}_{\psi} g_{\mathrm{bc}}(\gamma)=0
$$

The initial data

$$
A^{\mathrm{a}}=\psi^{\mathrm{a}}(\gamma, \gamma), \quad B^{\mathrm{ab}}=\nabla^{\mathrm{a}} \psi^{\mathrm{b}}(\gamma, \gamma)
$$

determine $\psi^{a}(x, \gamma)$ throughout $N$. In Minkowski spacetime, one finds that

$$
\psi^{\alpha}=A^{\alpha}+(x-\gamma)^{\beta} B_{\beta}{ }^{\alpha}
$$

in the usual coordinates. These coincide exactly with all of the affine collineations in this geometry.

In general, vector fields satisfying (3) in a curved spacetime also have the form (17) for some $A^{\text {a }}$ and $B^{\text {ab }}$. This is most easily seen by noting that vector fields with the given form have been obtained before as general solutions to the equation of geodesic deviation (also known as the Jacobi equation) 25]

$$
\sigma^{b} \sigma^{c}\left(\nabla_{b} \nabla_{c} \psi_{a}-R_{a b c}{ }^{d} \psi_{d}\right)=0 .
$$

For any fixed $x$, this is an ordinary differential equation along the geodesic connecting that point to $\gamma$. Solving it repeatedly for all geodesics in $N$ passing through this origin reproduces the vector fields (17). It is clear that such solutions always exist as long as the geometry is reasonably smooth. These are the spacetime's Jacobi fields about $\gamma$. The bitensors $H^{a}{ }_{\mathrm{a}} \sigma^{\mathrm{a}}{ }_{\mathrm{b}}$ and $H^{a}{ }_{\mathrm{a}} \sigma_{\mathrm{b}}$ are known as Jacobi propagators.

Solutions to the geodesic deviation equation effectively map one geodesic into another while preserving the affine parameters of both curves. It was noted above that this is the defining characteristic of affine collineations. The difference is that such vector fields must map every geodesic into another geodesic. This intuitive argument makes it clear that all affine collineations - or Killing fields as special cases - must be solutions of (21). The proof follows from noting that second derivatives of any exact affine collineation $Y_{\mathrm{A}}^{a}(x)$ must satisfy

$$
\nabla_{b} \nabla_{c} Y_{\mathrm{A}}^{a}=-R_{b d c}{ }^{a} Y_{\mathrm{A}}^{d} .
$$

This result is clear from (A.12), and is actually equivalent to (3). Substituting it into (21) shows that all affine collineations are indeed special cases of Jacobi fields. As expected, $Y_{\mathrm{A}}^{a}$ satisfies the geodesic deviation equation along all geodesics; even those that do not pass through $\gamma$. This point illustrates precisely how (21) generalizes the equation defining an affine collineation. It is simply (22) contracted into $\sigma^{b} \sigma^{c}$. Alternatively, the Jacobi equation is equivalent to (A.8).

To summarize, the following is now evident: 
Theorem 1 Let $N$ be a normal neighborhood of some point $\gamma$. Define a Jacobi field $\psi^{a}(x, \gamma)$ to be a solution of (21) throughout this region. It is explicitly given by (17) for some initial data with the form (19). The set of all Jacobi fields about a fixed $\gamma$ forms a 20-dimensional group in four spacetime dimensions. Each element satisfies $\mathcal{L}_{\psi} \nabla=0$ at $\gamma$. Furthermore, all affine collineations are members of this group.

These properties motivate our identification of the Jacobi fields as generalizations of affine collineations near $\gamma$.

Further results that strengthen this decision are derived in the appendix. Even though few Jacobi fields are genuine affine collineations, all can be interpreted as exact symmetries of certain quantities connected with the spacetime's geometric structure:

Theorem 2 Given a Jacobi field defined as in theorem 1, it is always true that

$$
\mathcal{L}_{\psi} \sigma^{\mathrm{a}}=\mathcal{L}_{\psi} \sigma^{a}=\mathcal{L}_{\psi} \sigma^{\mathrm{a}}{ }_{a}=\mathcal{L}_{\psi} H^{a}{ }_{\mathrm{a}}=0,
$$

where one of the arguments in each of these equations is taken to be the origin $\gamma$.

Lie derivatives on two-point tensor fields are defined to act independently on each argument. See (A.1), for example. Quantities appearing in this theorem are all important in Riemann normal coordinate systems parameterizing arbitrary points $x$ by the components of $X^{\mathrm{a}}=-\sigma^{\mathrm{a}}(x, \gamma)$. In terms of a more direct interpretation of the Jacobi fields as approximately satisfying (3), Lie derivatives of the metric with respect to an arbitrary $\psi^{a}$ are strongly constrained by the identities (A.7)-(A.10).

Statements of this sort do not exhaust the connections between the Jacobi equation and a spacetime's symmetries. There is a sense in which higher-rank Killing tensors that may exist are also solutions to the geodesic deviation equation [26]. Furthermore, projective collineations can be shown solve an inhomogeneous form of (21) proportional to $\sigma_{a}$ [27. These observations will not be discussed any further here, although it is possible that they could be used to generalize the present framework.

\subsection{Special cases}

It is often useful to single out a subset of the Jacobi fields distinguished by antisymmetric $B_{\mathrm{ab}}=\nabla_{\mathrm{a}} \psi_{\mathrm{b}}$. These may be said to generalize only the Killing fields of a given spacetime. Distinguishing them with a subscript "K," they clearly satisfy $\mathcal{L}_{\psi_{\mathrm{K}}} g_{\mathrm{ab}}(\gamma)=0$ as well as (18). Such objects form a 10-dimensional group that may be thought of as a generalization of the Poincaré group. They have been suggested before as useful generators for the linear and angular momenta of extended matter distributions [25, 28, 29. Fixing a hypersurface $\Sigma$ that passes through $\gamma$ and the worldtube of some well-behaved spatially-compact stress-energy distribution $T^{a b}$, let

$$
p_{\mathrm{a}}(\gamma, \Sigma) A^{\mathrm{a}}+\frac{1}{2} S_{\mathrm{ab}}(\gamma, \Sigma) B^{[\mathrm{ab}]}=\int_{\Sigma} T^{a}{ }_{b} \psi_{\mathrm{K}}^{b} \mathrm{~d} S_{a} .
$$

Varying the 10 free parameters here determines the four linear momenta $p^{\mathrm{a}}$ and six angular momenta $S^{\text {ab }}=S^{[\mathrm{ab}]}$. Explicit formulae are easily found using (17). They coincide with standard definitions in flat spacetime (where all $\psi_{\mathrm{K}}^{a}$ are Killing).

Theorem 2 is easily expanded for these vector fields:

Corollary 1 Given any Jacobi field $\psi_{\mathrm{K}}^{a}$ satisfying $\mathcal{L}_{\psi_{\mathrm{K}}} g_{\mathrm{ab}}=0$,

$$
\mathcal{L}_{\psi_{\mathrm{K}}} \sigma=\mathcal{L}_{\psi_{\mathrm{K}}} \sigma_{\mathrm{a}}=\mathcal{L}_{\psi_{\mathrm{K}}} \sigma_{a}=\mathcal{L}_{\psi_{\mathrm{K}}} \sigma_{\mathrm{a} a}=\mathcal{L}_{\psi_{\mathrm{K}}} H^{a \mathrm{a}}=0 .
$$

Again, one argument in each of these equations is assumed to be $\gamma$. 
This follows from the well-known identity [23, 24]

$$
\sigma^{\mathrm{a}} \sigma_{\mathrm{a}}=\sigma^{a} \sigma_{a}=2 \sigma,
$$

and its first derivative

$$
\sigma^{\mathrm{a}}{ }_{a} \sigma^{a}=\sigma^{\mathrm{a}} \text {. }
$$

By definition, $\sigma=g_{\mathrm{ab}}(\gamma) X^{\mathrm{a}} X^{\mathrm{b}} / 2$ is one-half of the squared geodesic distance between $\gamma$ and $x$. Killing-type Jacobi fields based at $\gamma$ therefore drag both arguments of $\sigma(x, \gamma)$ in such a way that distances are preserved.

It is also possible to identify Jacobi fields that act like homotheties near $\gamma$. These are distinguished by letting

$$
B_{(\mathrm{ab})}=\frac{1}{2} \mathcal{L}_{\psi_{\mathrm{H}}} g_{\mathrm{ab}}(\gamma)=c g_{\mathrm{ab}}(\gamma) .
$$

As in (2), $c$ is an arbitrary constant. For simplicity, the purely Killing components of some prospective $\psi_{\mathrm{H}}^{a}$ can be removed by setting $A_{\mathrm{a}}=B_{[\mathrm{ab}]}=0$ and $c \neq 0$. Substitution into (17) then shows that

$$
\psi_{\mathrm{H}}^{a}=-c H^{a}{ }_{\mathrm{a}} \sigma^{\mathrm{a}}=c \sigma^{a} .
$$

This second equality follows from contracting $\delta_{b}^{a}=-H^{a}{ }_{a} \sigma^{\mathrm{a}}{ }_{b}$ with $\sigma^{b}$ and using (27).

The simplicity of (29) is interesting, although perhaps not surprising. It is consistent with the interpretation of $-\sigma^{\mathrm{a}}$ as a "separation vector" between $x$ and $\gamma$. As has been noted before, $\sigma^{\mathrm{a}}{ }_{b} \rightarrow-\delta_{\beta}^{\alpha}$ in a normal coordinate system. The components of $\sigma^{a}$ would therefore be equal to $X^{\alpha}$. The normal coordinate functions themselves act as components of an approximately homothetic vector field. This is unique up to a constant factor and the addition of Killing-type Jacobi fields. It generalizes the dilations of flat spacetime.

Generalized Killing tensors of various types can also be generated from Jacobi fields. In analogy to (4), let

$$
\mathcal{K}_{a b}=\mathcal{L}_{\psi} g_{a b} .
$$

These objects exactly satisfy (5) at $\gamma$, and presumably approximate it near this point. It is straightforward to write down other objects which also have this property. For example, two (possibly identical) Killing-type Jacobi fields $\psi^{a}$ and $\bar{\psi}^{a}$ can be used to define

$$
\mathcal{K}_{a b}^{\prime}=\psi_{(a} \bar{\psi}_{b)} .
$$

This expression clearly generalizes to approximate Killing tensors of any rank. Exact second-rank Killing tensors probably exist that cannot be written in either of these forms, so it is unclear how useful they are.

Very near $\gamma$, it is possible to approximate the Jacobi fields explicitly. This will be especially useful in Sect. 4 4 below, where a notion of gravitational current is introduced with respect to a given vector field. Consider a Taylor expansion of $\mathcal{L}_{\psi} g_{a b}$ in powers of $X^{\mathrm{a}}$. The first two terms in this series are trivially obtained from (18) and (19). Better approximations involve third and higher derivatives of $\mathcal{L}_{\psi} g_{a b}$ in the limit $x \rightarrow \gamma$. The lowest order interesting terms can be found from (A.19) and (A.24). Making use of (A.18), the final results are that

$$
\mathcal{L}_{\psi} g_{a b} \simeq \sigma^{\mathrm{a}}{ }_{a} \sigma^{\mathrm{b}}{ }_{b}\left[\mathcal{L}_{\psi} g_{\mathrm{ab}}-\frac{1}{3} X^{\mathrm{c}} X^{\mathrm{d}}\left(\mathcal{L}_{\psi} R_{\mathrm{acbd}}+\frac{1}{2} X^{\mathrm{f}} \mathcal{L}_{\psi} \nabla_{\mathrm{f}} R_{\mathrm{acbd}}\right)\right]+\mathrm{O}\left(X^{4}\right),
$$


and

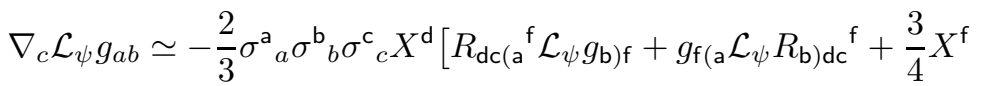

$$
\begin{aligned}
& \left.\times\left(\frac{1}{3} g_{\mathrm{h}\left({ }_{\mathrm{a}}\right.} \mathcal{L}_{\psi} \nabla^{\mathrm{h}} R_{\mathrm{b}) \mathrm{dfc}}-g_{\mathrm{ha}} \mathcal{L}_{\psi} \nabla_{\mathrm{d}} R_{\mathrm{f}(\mathrm{bc})}{ }^{\mathrm{h}}-g_{\mathrm{hb}} \mathcal{L}_{\psi} \nabla_{\mathrm{d}} R_{\mathrm{f}(\mathrm{ac})}{ }^{\mathrm{h}}\right)\right]+\mathrm{O}\left(X^{3}\right) .
\end{aligned}
$$

Lie derivatives here are evaluated at $\gamma$, so they only involve $A_{\mathrm{a}}, B_{\mathrm{ab}}, g_{\mathrm{ab}}, R_{\mathrm{abcd}}$, and its first two derivatives. The factors of $\sigma^{a}{ }_{a}$ in front of these equations are used as a convenient means for converting tensors at $\gamma$ into tensors at $x$. It is perhaps more typical to use parallel propagators $g^{\mathrm{a}}{ }_{a}$ for this purpose 24, although the aforementioned simplicity of $\sigma_{a}^{\mathrm{a}}{ }_{a}$ in normal coordinates makes it an attractive alternative. There is very little difference at low orders regardless. $\sigma^{\mathrm{a}}{ }_{a}$ can be freely interchanged with $-g^{\mathrm{a}}{ }_{a}$ in (33). This is also possible in (32) when $B_{(\mathrm{ab})}=0$.

Approximations like these are not useful over regions where the curvature changes significantly, or on length scales approaching the curvature radius. An alternative approach is to expand the various bitensors built from $\sigma$ using its definition as an integral along a geodesic. Simplifications can often be introduced by ignoring all terms nonlinear in the Riemann tensor. A general method for this type of weak-field procedure may be found in 23,30 . Specific details involved with expanding the Jacobi fields in this way will not be given here.

\section{Symmetries near a worldline}

The Jacobi fields just discussed generalize the idea of a Killing field or more general affine collineation in a normal neighborhood of a given point. This is useful for some purposes, although it does not have a very clear physical interpretation. The choice of origin should presumably correspond to a preferred point, although there are few of these that might arise in practice. It is often more useful to base the idea of an approximate symmetry off of a given timelike worldline rather than a single point. This could correspond to the path of some observer. In some cases, the physical system picks out preferred reference frames. A binary star system experiencing no mass transfer can admit three center-of-mass frames (rigorously defined in [25, 31, 35]), for example. Two of these are associated with the individual stars, while the third describes the system as a whole. There are also preferred observers in most cosmological models. Expressing a system's dynamics in terms of quantities associated with these frames has an obvious physical interpretation. The distinction between approximate symmetries defined with respect to a point versus a worldline is closely analogous to the one between Riemann and Fermi normal coordinate systems.

The concept of an observer here will be taken to mean a timelike worldline $\Gamma$ together with a set of hypersurfaces $\Sigma(s)$ that foliate a surrounding worldtube $W$. It will be assumed that each of these hypersurfaces is a normal neighborhood of the point $\gamma(s)$ where it intersects the central worldline. Each of them is therefore formed by a collection of radially-emanating geodesics of (usually) finite length. The most typical examples would be the past-directed null geodesics or the spacelike set orthogonal to $\dot{\gamma}^{\mathrm{a}}=\mathrm{d} \gamma^{\mathrm{a}} / \mathrm{d} s$ at $\gamma(s)$. Other choices are possible, however. Regardless, a worldline and foliation together will be referred to as an observer's reference frame. 


\subsection{A family of Jacobi fields}

Symmetries adapted to a particular frame can be constructed using a one-parameter family of Jacobi fields $\psi^{a}(x, \gamma(s))$. Any such family is fixed by specifying $A_{\mathrm{a}}(s)$ and $B_{\mathrm{ab}}(s)$ as defined in (19). An optimal way of connecting initial data between different points on $\Gamma$ therefore must be found. Before considering this problem, it is first useful to collapse the family of Jacobi fields into an ordinary vector field $\xi^{a}(x)$. Let $\tau(x)$ be defined so as to identify which leaf of the foliation includes an arbitrary point $x$ in the worldtube $W$. More concisely, it always satisfies $x \in \Sigma(\tau(x))$. The assumption that each hypersurface is a normal neighborhood of an appropriate point on $\Gamma$ implies that $\tau$ is always single-valued. Now set

$$
\xi^{a}(x)=\psi^{a}(x, \gamma(\tau(x))) .
$$

The generalized affine collineations (GACs) to be defined below will be of this form for a particular class of families $\psi^{a}(x, \gamma(s))$.

One potential application for a generalized symmetry constructed using a particular frame is in the definition of quantities that might be approximately conserved as one moves along $\Gamma$. As an example, consider integrals of conserved stress-energy tensors similar to (24). One might define the component of momentum generated by a $\xi^{a}$ of the form (34) to be

$$
\mathcal{P}_{\xi}(s)=\int_{\Sigma(s)} T_{b}^{a} \xi^{b} \mathrm{~d} S_{a}
$$

The evolution of this quantity crucially depends on how the parameters $A_{\mathrm{a}}(s)$ and $B_{\mathrm{ab}}(s)$ in (19) are connected along $\Gamma$. It is well-known that $\mathcal{P}_{\xi}$ is conserved if $\xi^{a}$ is Killing and no matter flows across the boundary of the worldtube. In this case, initial data for the one-parameter family of Jacobi fields must satisfy the Killing transport $(\mathrm{KT})$ equations on $\Gamma$ :

$$
\begin{aligned}
& \mathrm{D} A_{\mathrm{a}} / \mathrm{d} s=\dot{\gamma}^{\mathrm{b}} B_{\mathrm{ba}} \\
& \mathrm{D} B_{\mathrm{ab}} / \mathrm{d} s=-R_{\mathrm{abc}}{ }^{\mathrm{d}} \dot{\gamma}^{\mathrm{c}} A_{\mathrm{d}} .
\end{aligned}
$$

If there exists an exact Killing vector $Y_{\mathrm{K}}^{a}$ such that $A^{\mathrm{a}}=Y_{\mathrm{K}}^{\mathrm{a}}$ and $B^{\mathrm{ab}}=\nabla^{\mathrm{a}} Y_{\mathrm{K}}^{\mathrm{b}}$ at a given $s=s_{0}$, relations like these will hold for all $s$. Furthermore, momenta $p^{\mathrm{a}}$ and $S^{\mathrm{ab}}$ identified using (24) would satisfy

$$
0=\left(\dot{p}_{\mathrm{a}}-\frac{1}{2} S^{\mathrm{bc}} R_{\mathrm{bcda}} \dot{\gamma}^{\mathrm{c}}\right) Y_{\mathrm{K}}^{\mathrm{a}}+\frac{1}{2}\left(\dot{S}_{\mathrm{ab}}-2 p_{[\mathrm{a}} \dot{\gamma}_{\mathrm{b}]}\right) \nabla^{\mathrm{a}} Y_{\mathrm{K}}^{\mathrm{b}}
$$

If there were a full complement of ten Killing vectors, all possible versions of this expression would together be equivalent to the Papapetrou equations. More generally, Papapetrou's result is only an approximation. Any deviations can be understood using a general 10-parameter family of possibly approximate isometries. One might expect these corrections to be minimized if $A_{\mathrm{a}}$ and $B_{\mathrm{ab}}$ always satisfy the KT equations even when no exact Killing fields exist.

Definition 1 Let a generalized affine collineation $(G A C) \xi^{a}(x)$ associated with a reference frame $\{\Gamma, \Sigma\}$ be derived from a family of Jacobi fields via (34). Individual elements of the family and their first derivatives satisfy the Killing transport equations (36a) and (36b) on $\Gamma$.

Although this definition was motivated by the properties of conserved momenta in very particular spacetimes, it also arises from much more general (if less physical) 
arguments. Consider all possible initial data for vectors built from Jacobi fields using (34). It is reasonable to suppose that any GAC should be exactly affine on $\Gamma$; i.e.

$$
\left.\nabla_{\mathrm{a}} \mathcal{L}_{\xi} g_{\mathrm{bc}}\right|_{\Gamma}=0 .
$$

It can also be expected that $A_{\mathrm{a}}$ and $B_{\mathrm{ab}}$ fix $\xi^{a}$ and its first derivatives on $\Gamma$ just as they do for $\psi^{a}$. Generalizing (19), let

$$
A^{\mathrm{a}}(s)=\xi^{\mathrm{a}}(\gamma(s)), \quad B^{\mathrm{ab}}(s)=\nabla^{\mathrm{a}} \xi^{\mathrm{b}}(\gamma(s)) .
$$

We start with the second of these constraints. Directly differentiating (34) implies that

$$
\mathcal{L}_{\xi} g_{a b}=\mathcal{L}_{\psi} g_{a b}+2 \dot{\psi}_{(a} \nabla_{b)} \tau,
$$

where the Lie derivative with respect to $\psi^{a}(x, \tau)$ on the right-hand side is understood (as usual) to involve only the first argument of this vector field. It will be assumed that the foliation is always sufficiently smooth that derivatives of $\tau$ remain well-defined throughout $W$, and on $\Gamma$ in particular. Evaluating (40) on the central worldline requires knowledge of $\dot{\psi}^{a}(\gamma, \gamma)$. Coincidence limits like these are commonly denoted with brackets. For example,

$$
\left[\dot{\psi}^{a}\right](\gamma)=\lim _{x \rightarrow \gamma} \frac{\partial}{\partial s} \dot{\psi}^{a}(x, \gamma(s)) .
$$

The convention of using different fonts for indices referring to $x$ and $\gamma$ cannot be consistently applied in expressions like this. No confusion should arise, however. Limits like (41) are easily computed using Synge's rule [23, 24]. In this case,

$$
\begin{aligned}
{\left[\dot{\psi}^{a}\right] } & =\dot{\gamma}^{\mathrm{b}}\left[\nabla_{\mathrm{b}} \psi^{a}\right] \\
& =\dot{\gamma}^{\mathrm{b}}\left(\nabla_{\mathrm{b}}\left[\psi^{a}\right]-\delta_{\mathrm{b}}^{b}\left[\nabla_{b} \psi^{a}\right]\right) \\
& =\mathrm{D} A^{a} / \mathrm{d} s-\dot{\gamma}^{\mathrm{b}} B_{\mathrm{b}}{ }^{a} .
\end{aligned}
$$

It follows that (39) holds for all initial data iff (36a) is satisfied.

The other KT equation arises from enforcing (38). Noting (18) and (36a), it must be true that

$$
\left[\nabla_{a} \dot{\psi}^{b}\right]=0 .
$$

$\left[\ddot{\psi}^{a}\right]$ also has to vanish, although this term is equal to $-\dot{\gamma}^{b}\left[\nabla_{b} \dot{\psi}^{a}\right]$. Requiring (43) is therefore sufficient. Using the same type of procedure as in (42) shows that

$$
\left[\nabla_{a} \dot{\psi}_{b}\right]=\mathrm{D} B_{a b} / \mathrm{d} s+R_{a b c}{ }^{d} \dot{\gamma}^{c} A_{d}
$$

Deriving this is straightforward other than noting that (22) - although mentioned for exact affine collineations - also holds for any Jacobi field at its origin. Regardless, the conclusion is that the second Killing transport equation (36b) ensures that $\nabla_{\mathrm{a}} \mathcal{L}_{\xi} g_{\mathrm{bc}}$ vanishes everywhere on $\Gamma$.

Noting that the KT equations have the same significance for general affine collineations as they do for ordinary Killing fields [15, it easily follows that

Theorem 3 The class of all generalized affine collineations associated with a given reference frame forms a 20-dimensional group in four spacetime dimensions. Every GAC satisfies $\mathcal{L}_{\xi} \nabla=0$ on $\Gamma$, and all exact affine collineations are members of this class. 
This is closely related to theorem 1. It strongly supports definition 1 and the intuitive identification of GACs with approximate symmetries inside $W$.

At least in principle, finding GACs associated with a particular reference frame is straightforward. Suppose that $A_{\mathrm{a}}\left(s_{0}\right)$ and $B_{\mathrm{ab}}\left(s_{0}\right)$ are given as initial data at some $\gamma_{0}=\gamma\left(s_{0}\right)$. The goal is then to determine the $\xi^{a}(x)$ satisfying (39) at the appropriate point. This is done by first applying the KT equations to the given parameters along $\Gamma$ from $\gamma_{0}$ to $\gamma(\tau(x))$. The geodesic deviation equation (21) is then integrated between this latter point and $x$ using the initial conditions (19). Both of these operations simply require finding the solutions to well-behaved ordinary differential equations. Alternatively, $\xi^{a}$ could also be obtained using the explicit expression (17) together with the KT equations and (34).

Our prescription for generalizing arbitrary affine collineations may appear somewhat awkward. Killing transport equations are being applied along $\Gamma$, while the Jacobi equation is used on geodesics intersecting that worldline. These two procedures are not as different as they might appear. Trying to use Killing transport everywhere would generically lead to inconsistencies. Derivatives of the field expected from the KT equations would not usually match the derivatives computed from $\xi^{a}$ itself. Only the tangential components of these derivatives can be consistently fixed by integrating ordinary differential equations along a collection of radial geodesics. Weakening the Killing transport equations to take this into account exactly reproduces the geodesic deviation equation. This may be seen by rewriting (21) as a pair of first order differential equations on geodesics connecting $x$ to $\gamma(\tau(x))$. Denote the unit tangent vector to one such geodesic by $u^{a}(l)$. Also set $\hat{A}^{a}=\psi^{a}$ and $\hat{B}_{a b}=\nabla_{a} \psi_{b}$ everywhere. It is then straightforward to show that

$$
\begin{aligned}
& \mathrm{D} \hat{A}^{a} / \mathrm{d} l=u^{b} \hat{B}_{b a} \\
& u^{a} \mathrm{D} \hat{B}_{a b} / \mathrm{d} l=-R_{a b c} d u^{a} u^{d} \hat{A}_{d} .
\end{aligned}
$$

The first of these equations has exactly the same form as (36a), while the second is essentially (36b) contracted with $u^{a}$. Killing and Jacobi transport are therefore very closely related operations. The latter does not uniquely propagate $\hat{B}_{a b}$ from given initial data, so it is weaker. These remarks also clarify in what sense Jacobi fields or GACs approximate (3) or (22).

\subsection{Special cases and properties of GACs}

From a physical perspective, momenta like (35) should be definable even in the absence of any exact isometries. This is most conveniently done with a particular class of GACs that generalize only the Killing fields. In analogy to the Killing-type Jacobi fields discussed in Sect. 2.2, suppose that $B_{(\mathrm{ab})}$ vanishes on at least one point of $\Gamma$. It immediately follows from (36b) that it must actually vanish everywhere. The Killing-type GACs therefore form a 10-dimensional group of vector fields satisfying

$$
\left.\mathcal{L}_{\xi_{\mathrm{K}}} g_{\mathrm{ab}}\right|_{\Gamma}=0,
$$

as well as (38). They may be thought of as generalizing the Poincaré symmetries of flat spacetime near a given observer.

It also possible to single out GACs that are approximately homothetic in the sense that

$$
\left.\mathcal{L}_{\xi_{\mathrm{H}}} g_{\mathrm{ab}}\right|_{\Gamma}=2 c g_{\mathrm{ab}} .
$$


This requires setting $B_{(\mathrm{ab})}=c g_{\mathrm{ab}}$. While always possible, the remaining components of the initial data cannot be explicitly solved for except in the case when $\Gamma$ is a geodesic. It then self-consistent to choose $B_{[\mathrm{ab}]}=0$. The obvious way of doing this is to normalize $\dot{\gamma}^{\text {a }}$ to unity and set

$$
A_{\mathrm{a}}=c(s-\bar{s}) \dot{\gamma}_{\mathrm{a}}
$$

for some constant $\bar{s}$. It is easily verified that the given parameters satisfy the KT equations. One homothetic-type GAC associated with an affinely parameterized geodesic therefore has the form

$$
\xi_{\mathrm{H}}^{a}=c\left[\sigma^{a}+(\tau-\bar{s}) H^{a}{ }_{\mathrm{a}} \sigma^{\mathrm{a}}{ }_{\mathrm{b}} \dot{\gamma}^{\mathrm{b}}\right] .
$$

As usual, Killing-type Jacobi fields may be added to this without spoiling (47). It should also be emphasized that homothetic-type GACs are not restricted to geodesic frames. This is just the case where closed-form solutions of the KT equations can be obtained by inspection.

Many of the properties derived for Jacobi fields in Sect. 22 and the appendix can be carried over at least partially for the GACs. For example,

$$
\mathcal{L}_{\xi} \sigma^{\mathrm{a}}=\mathcal{L}_{\psi} \sigma^{\mathrm{a}}=0
$$

if the arguments are of the form $(x, \gamma(\tau(x)))$ and $\xi^{a}$ and $\psi^{a}$ are related via (34). This may be interpreted as stating that spatial Fermi coordinates are preserved under flows generated by $\xi^{a}$. Since the hypersurfaces $\Sigma(s)$ can be described as a set of geodesics intersecting $\gamma(\tau(x))$, it will always be true that $\sigma^{a} \nabla_{a} \tau=0$. This means that

Theorem 4 Given a general GAC $\xi^{a}, \mathcal{L}_{\xi} \sigma^{a}=\mathcal{L}_{\xi} \sigma^{a}=0$ when the arguments of these equations are as in (50). Killing-type GACs $\xi_{\mathrm{K}}^{a}$ also satisfy $\mathcal{L}_{\xi_{\mathrm{K}}} \sigma=\mathcal{L}_{\xi_{\mathrm{K}}} \sigma_{\mathrm{a}}=0$ with the same restriction.

The identity (A.7) serves to constrain Lie derivatives of the metric with respect to Jacobi fields. A direct analog of this equation for an arbitrary GAC would involve an additional term. Despite this, contracting the result with $\sigma^{b}$ leads to the simple conclusion

$$
\sigma^{a} \sigma^{b} \mathcal{L}_{\xi} g_{a b}=2 \sigma^{\mathrm{a}} \sigma^{\mathrm{b}} B_{(\mathrm{ab})} .
$$

"Purely radial" components of $\mathcal{L}_{\xi} g_{a b}$ therefore vanish for Killing-type GACs.

Many other results can be carried over in similar ways. One that is of particular interest is the behavior of $\mathcal{L}_{\xi} g_{a b}$ or $\nabla_{a} \mathcal{L}_{\xi} g_{b c}$ near $\Gamma$. As with the Jacobi fields, it is possible to see how close a GAC comes to being affine as its reference worldline is approached. Analogs of (32) and (33) may be obtained using expansions like (40) and the identity (A.12). Simplifying terms with the Killing transport equations, the lowest order correction to (32) is

$$
\mathcal{L}_{(\xi-\psi)} g_{a b} \simeq \frac{2}{3} \sigma^{\mathrm{a}}{ }_{a} \sigma^{\mathrm{b}}{ }_{b} \nabla_{(\mathrm{a}} \tau g_{\mathrm{b}) \mathrm{f}} \dot{\gamma}^{\mathrm{h}} X^{\mathrm{c}} X^{\mathrm{d}} \mathcal{L}_{\xi} R_{\mathrm{hcd}}{ }^{\mathrm{f}}+\mathrm{O}\left(X^{3}\right) .
$$

Similarly, the first interesting change to (33) has the form

$$
\begin{aligned}
\nabla_{a} \mathcal{L}_{(\xi-\psi)} g_{b c} \simeq- & \frac{4}{3} \sigma^{\mathrm{a}}{ }_{a} \sigma^{\mathrm{b}}{ }_{b} \sigma^{\mathrm{c}}{ }_{c} X^{\mathrm{d}} \dot{\gamma}^{\mathrm{f}}\left[g_{\mathrm{h}(\mathrm{b}} \nabla_{\mathrm{c})} \tau\left(\delta_{\mathrm{a}}^{\mathrm{l}}-\dot{\gamma}^{\mathrm{l}} \nabla_{\mathrm{a}} \tau\right) \mathcal{L}_{\xi} R_{\mathrm{f}(\mathrm{ld})}{ }^{\mathrm{h}}\right. \\
& \left.+\frac{1}{2} \nabla_{\mathrm{a}} \tau\left(R_{\mathrm{df}(\mathrm{b}}{ }^{\mathrm{h}} \mathcal{L}_{\xi} g_{\mathrm{c}) \mathrm{h}}-g_{\mathrm{h}(\mathrm{b}} \mathcal{L}_{\xi} R_{\mathrm{c}) \mathrm{fd}}{ }^{\mathrm{h}}\right)\right]+O\left(X^{2}\right) .
\end{aligned}
$$

As before, the magnitudes of these terms depend on how close $\xi^{a}$ is to being a symmetry of the Riemann tensor on the observer's worldline. 


\section{Mechanics and conservation laws}

It has already been remarked that one of the main applications of exact symmetries in physics is to the formulation of conservation laws. These take several forms. Perhaps the most basic are those associated with a spacetime's geodesics. It is sometimes possible for such curves to be at least partially parameterized by a number of constants associated with geometric symmetries. More interestingly, conservation laws can also be associated with extended matter distributions. Assuming only that stress-energy tensors satisfy

$$
\nabla_{a} T^{a b}=0
$$

tends to lead to the definition of slowly-varying parameters like those discussed in connection with (24) and (35). The situation becomes much more interesting in full general relativity. Einstein's equation implies stress-energy conservation, although it also connects symmetries of the geometry to those of the matter distribution (and vice versa). This allows the introduction of exact conservation laws in arbitrary spacetimes.

\subsection{Geodesics}

It is well-known that any Killing fields that may exist provide first integrals of the geodesic equation. These can be used both to derive and parameterize the geodesics of a given spacetime. While less commonly discussed, similar quantities can also be associated with other kinds of symmetries [19, 33. Unlike in the Killing vector case, the presence of more general collineations sometimes implies the existence of interesting conserved quantities that are not linear in the geodesic's four-velocity. The curve's affine parameter can also appear explicitly. As a direct calculation will easily verify, two constants associated with an exact affine collineation $Y_{\mathrm{A}}^{a}$ are

$$
\begin{aligned}
& C_{1}=\dot{y}^{a} \dot{y}^{b} \mathcal{L}_{Y_{\mathrm{A}}} g_{a b} \\
& C_{2}=\dot{y}_{a} Y_{\mathrm{A}}^{a}-\frac{1}{2} l C_{1} .
\end{aligned}
$$

These quantities remain fixed along any affinely-parameterized geodesic $y(l)$. The first becomes degenerate if $Y_{\mathrm{A}}^{a}$ is Killing. $C_{2}$ then reduces to the standard conserved quantity associated with a Killing field. Other constants can sometimes be written down by combining $Y_{\mathrm{A}}^{a}$ with an exact Killing tensor [19, 32. Such constructions will not be discussed here.

Consider instead expressions like those just given with $Y_{\mathrm{A}}^{a}(x)$ replaced by some Jacobi field $\psi^{a}(x, \gamma)$. We then have

$$
\dot{C}_{1}=\frac{\mathrm{d} C_{1}}{\mathrm{~d} l}=-\frac{2}{l} \frac{\mathrm{d} C_{2}}{\mathrm{~d} l}=\dot{y}^{a} \dot{y}^{b} \dot{y}^{c} \nabla_{(a} \mathcal{L}_{\psi} g_{b c)} .
$$

The tangent vectors are proportional to $\sigma^{a}(y, \gamma)$ for the special case of geodesics passing through $\gamma$. It then follows from (A.10) that both $C_{1}$ and $C_{2}$ remain conserved along all such trajectories. Each Jacobi field generates exact geodesic constants in this way. In terms of the initial data $A_{\mathrm{a}}$ and $B_{\mathrm{ab}}$, these have the values

$$
C_{1}=2 \dot{y}^{\mathrm{a}} \dot{y}^{\mathrm{b}} B_{(\mathrm{ab})}, \quad C_{2}=\dot{y}^{\mathrm{a}} A_{\mathrm{a}}
$$

when the parameter $l$ is chosen to vanish at $\gamma$. It is clear from this that $B_{[\mathrm{ab}]}$ is irrelevant. Multiple Jacobi fields may therefore generate the same constants on a particular curve. 
Exact affine collineations generalize these results by also applying to non-radial geodesics. Expansions like (33) can be used to derive how close general Jacobi fields come to this ideal. To lowest nonvanishing order,

$$
\dot{C}_{1} \simeq-\frac{4}{3}\left(\dot{y}^{a} \sigma^{\mathrm{a}}{ }_{a}\right)\left(\dot{y}^{b} \sigma^{\mathrm{b}}{ }_{b}\right)\left(\dot{y}^{c} \sigma^{\mathrm{c}}{ }_{c}\right) X^{\mathrm{d}} R_{\mathrm{dbc}}{ }^{\mathrm{f}} \mathcal{L}_{\psi} g_{\mathrm{af}}+\mathrm{O}\left(X^{2}\right) .
$$

This term vanishes if $B_{(\mathrm{ab})}=0$, so the Killing-type Jacobi fields usually provide more accurate "conservation laws" for arbitrary geodesics near $\gamma$. In these cases, $\dot{C}_{1}(l)$ scales like $(X / \mathcal{R})^{2} / \mathcal{R}$, where $\mathcal{R}$ is a curvature radius. This is a worst-case estimate. $C_{1}$ and $C_{2}$ will probably vary much more slowly if there is a physical sense in which the system is approximately symmetric. Rates of change for $C_{1}$ or $C_{2}$ can also be constrained using identities like (A.8). This effectively restricts how much these parameters can vary as a geodesic moves away from $\gamma$. More of their changes tend to occur as $y(l)$ moves across rather than with the radial geodesics.

Parameters like $C_{1}$ and $C_{2}$ can also be defined with respect to a $\mathrm{GAC} \xi^{a}$. These should remain approximately conserved for geodesics near an observer's worldline rather than geodesics near a point. They are exact constants for curves passing through $\Gamma$ along the reference foliation. This can be seen by using (40) to show that

$$
\dot{y}^{a} \dot{y}^{b} \dot{y}^{c} \nabla_{a} \mathcal{L}_{\xi} g_{b c}=\dot{y}^{a} \dot{y}^{b} \dot{y}^{c} \nabla_{a} \mathcal{L}_{\psi} g_{b c}
$$

when $\dot{y}^{a} \nabla_{a} \tau=0$. Values of $C_{1}$ and $C_{2}$ here are the same as in (57) if the quantities in that equation are evaluated at the point where $y$ intersects $\Gamma$. This might be useful in a coordinate system constructed from some collection of GACs. Alternatively, it can be viewed as a generalization of standard results near a given observer.

Further methods of parameterizing geodesics may be found by generalizing the constants associated with higher-rank Killing tensors. Given any exact Killing tensor $K_{a_{1} \cdots a_{n}}=K_{\left(a_{1} \cdots a_{n}\right)}$, the scalar

$$
C_{K}=K_{a_{1} \cdots a_{n}} \dot{y}^{a_{1}} \cdots \dot{y}^{a_{n}}
$$

is conserved along all geodesics $y(l)$. An analog of this quantity for the approximate Killing tensor (30) is exactly $C_{1}$ defined above. Something more interesting can be generated by substituting (31) into (60). In the second-rank case, one may define

$$
C_{K}=\left(\dot{y}_{a} \psi_{\mathrm{K}}^{a}\right)\left(\dot{y}_{b} \bar{\psi}_{\mathrm{K}}^{b}\right)
$$

for some Killing-type Jacobi fields $\psi_{\mathrm{K}}^{a}$ and $\bar{\psi}_{\mathrm{K}}^{a}$. This is easily generalized to involve an arbitrary number of products, although it is only interesting to consider linearly independent collections of Jacobi fields. The maximum number of useful products is therefore ten. All of these can be generated just from individual terms of the form $\dot{y}_{a} \psi_{\mathrm{K}}^{a}$. These are interpreted as approximate constants associated with objects that are nearly first-rank Killing tensors (i.e. Killing vectors). Everything of interest here can therefore be derived from the behavior of

$$
C_{3}=\dot{y}_{a} \psi_{\mathrm{K}}^{a}=C_{2}+\frac{1}{2} l C_{1}
$$

Although this depends only on the two approximate constants defined before, it may be interpreted as an additional useful parameter. It has the interesting property that

$$
\dot{C}_{3}=\frac{1}{2} C_{1} \text {. }
$$

Time derivatives do not appear on the right hand side. Consider the special case of a geodesic that passes through $\gamma$. Since the Jacobi field was assumed to be Killing at 
its origin, $C_{1}=0$. This is true everywhere, so $C_{3}$ also remains fixed along the entire geodesic. It actually coincides with $C_{2}$ in this case.

Differences arise when considering non-radial geodesics. It was remarked above that there was a sense in which $C_{1}$ and $C_{2}$ only varied due to non-radial components of $\dot{y}^{a}$. This type of statement can be made much more precise for $C_{3}$. Let

$$
\dot{y}^{a}(l)=u_{\|}(l) \sigma^{a}(y(l), \gamma)+u_{\perp}^{a}(l),
$$

where $u_{\perp}^{[a} \sigma^{b]}=0$. Now (4.7), (55a), and (63) show that

$$
\dot{C}_{3}=\frac{1}{2} u_{\perp}^{a} u_{\perp}^{b} \mathcal{L}_{\psi} g_{a b}
$$

This result is exact for all geodesics $y(l)$. There are many cases where $u_{\perp}^{a}$ becomes vanishingly small as $l \rightarrow \pm \infty$ (when the geodesic exists for these parameter values), so (65) provides a strong restriction on how much $C_{3}$ can vary in any given situation. Very near $\gamma$, (32) can be used to show that

$$
\dot{C}_{3} \simeq-\frac{1}{6}\left(u_{\perp}^{a} \sigma_{a}^{\mathrm{a}}{ }_{a}\right)\left(u_{\perp}^{b} \sigma^{\mathrm{b}}{ }_{b}\right) X^{\mathrm{c}} X^{\mathrm{d}} \mathcal{L}_{\psi} R_{\mathrm{acbd}}+\mathrm{O}\left(X^{3}\right) .
$$

The lowest order contributions here scale like $(X / \mathcal{R})^{2} / \mathcal{R}$. This is similar to the result expected for $\dot{C}_{1}$ when computed using a Killing-type Jacobi field.

\subsection{Extended matter distributions}

From a physical perspective, it is often more interesting to consider possibly approximate integrals of the equations of motion describing an extended matter distribution rather than a pointlike test particle. Suppose that this matter is modeled by a conserved stress-energy tensor $T^{a b}$. Contracting it with any exact Killing vectors that may exist yields conserved currents. These are equivalent to some subset of the typical laws of linear and angular momentum conservation known in flat spacetime. More generally, (54) shows that

$$
\nabla_{a}\left(T^{a}{ }_{b} Y^{b}\right)=\frac{1}{2} T^{a b} \mathcal{L}_{Y} g_{a b} .
$$

This holds for any vector field $Y^{a}$, although it is convenient to assume that it is a Killing-type GAC. The source term on the right-hand side may then be considered small near $\Gamma$. This therefore serves as an approximate conservation law. As long as there is no matter flow through $\partial \Sigma$, quantities like (35) might be expected to vary slowly in time.

Stress-energy conservation can be seen as a consequence of the diffeomorphism invariance of a system's underlying action. Constructions that are based on it are therefore useful in many theories of gravity besides general relativity. They can also hold for test bodies in fixed background geometries. This generality is quite restrictive. Much more can be said if the full Einstein equation is assumed to hold. Symmetries in the geometry are then related to symmetries in the matter fields. The presence of an exact Killing field $Y_{\mathrm{K}}^{a}$ that also satisfies $\mathcal{L}_{Y_{\mathrm{K}}} T_{a b}=0$ allows many interesting results to be proven regarding the momenta $p_{\mathrm{a}}$ and $S_{\mathrm{ab}}$ defined in (24). For example, the net force and torque on a body can be written explicitly in terms of the Killing field and its first derivative at a point. If $Y_{\mathrm{K}}^{a}$ is timelike, a body's center-of-mass can be shown to follow one of its orbits. Furthermore, mass centers always lie on the central axis of cylindrically symmetric spacetimes [12, 34. 
Momenta defined in terms of $T^{a}{ }_{b} \xi^{b}$ are useful for many purposes, although they are not conserved in the absence of exact Killing fields. Determining how they vary over time requires detailed knowledge of a body's internal structure. Alternative definitions for the linear and angular momenta of an extended body arise when using the full Einstein equation rather than just (54). Taking the trace of Ricci's identity and rearranging terms shows that

$$
R_{b}^{a} Y^{b}=\frac{1}{2}\left(g^{a c} g^{b d}-g^{a b} g^{c d}\right) \nabla_{b} \mathcal{L}_{Y} g_{c d}+\nabla_{b} \nabla^{[a} Y^{b]} .
$$

This holds for any vector field $Y^{a}$. Note that the second term on the right-hand side is always conserved. It follows that

$$
\nabla_{a}\left[2\left(T_{b}^{a} Y^{b}-\frac{1}{2} Y^{a} T_{b}^{b}\right)+j_{Y}^{a}\right]=0,
$$

where the "gravitational current" $j_{Y}^{a}$ associated with $Y^{a}$ has been defined by

$$
j_{Y}^{a}=\frac{1}{8 \pi}\left(g^{a b} g^{c d}-g^{a c} g^{b d}\right) \nabla_{b} \mathcal{L}_{Y} g_{c d}
$$

It clearly vanishes if $Y^{a}$ is an exact affine collineation. This is not the only case where the current's contribution to (69) disappears. Using the Bianchi identity,

$$
\nabla_{a} j_{Y}^{a}=-\frac{1}{8 \pi} g^{a b} \mathcal{L}_{Y} R_{a b}
$$

Any vector field satisfying $g^{a b} \mathcal{L}_{Y} R_{a b}=0$ will therefore generate conserved matter currents involving only $T_{b}^{a} Y^{b}-Y^{a} T_{b}^{b} / 2$. That such "contracted Ricci collineations" generate conservation laws for matter distributions has been noted before in [20, 21].

The viewpoint here will be to apply (68) and (69) with $Y^{a}$ replaced by some approximate symmetry.

Definition 2 Fix some family of Jacobi fields $\psi^{a}(x, \gamma(s))$ that generates a GAC via (34). Define the generalized Komar momentum $\mathcal{P}_{\psi}^{*}$ associated with these fields by

$$
\mathcal{P}_{\psi}^{*}(s)=\frac{1}{8 \pi} \oint_{\partial \Sigma(s)} \nabla^{[a} \psi^{b]} \mathrm{d} S_{a b} .
$$

As the name suggests, this has the same form and interpretation as a Komar integral. It is convenient to assume that $s$ is a fixed parameter for the purpose of evaluating the derivative in (72). Directly using a GAC in place of $\psi^{a}$ would add a dependence on the reference frame. Note that no such distinctions had to be made for the $\mathcal{P}_{\xi}$ defined in (35). Applying Stokes' theorem together with (68) and (70) shows that

$$
\mathcal{P}_{\psi}^{*}=\int_{\Sigma}\left[2\left(T_{b}^{a} \psi^{b}-\frac{1}{2} \psi^{a} T_{b}^{b}\right)+j_{\psi}^{a}\right] \mathrm{d} S_{a} .
$$

There is a well-defined sense in which changes in this quantity are determined by a combination of "gravitational wave" and matter fluxes across the boundary $\partial \Sigma$. In this interpretation, the amount of $\mathcal{P}_{\psi}^{*}$ carried away from a system via gravitational waves vanishes if the GAC associated with $\psi^{a}$ is an affine collineation.

The 20-parameter family of scalars $\mathcal{P}_{\psi}^{*}$ is intended to define the linear and angular momenta of an extended body. This is at least the interpretation for the 10-parameter subset satisfying $B_{(\mathrm{ab})}=0$. As in (24), it is possible to write these momenta in the more conventional form of tensor fields on $\Gamma$. Let

$$
\mathcal{P}_{\psi}^{*}=p_{\mathrm{a}}^{*} A^{\mathrm{a}}+\frac{1}{2} S_{\mathrm{ab}}^{*} B^{\mathrm{ab}}
$$


for all $A_{\mathrm{a}}$ and $B_{\mathrm{ab}}$. As written, the generalized angular momentum $S_{\mathrm{ab}}^{*}$ needn't have any particular index symmetries. Non-Killing Jacobi fields generate the symmetric components of this tensor, although such generality isn't necessary. Varying among all combinations of initial data completely recovers $p_{\mathrm{a}}^{*}$ and $S_{\mathrm{ab}}^{*}$. Direct expressions can also be obtained with the use of (17). Continuing to work with the less explicit form (174), rates of change of the tensor momenta may easily be extracted from $\dot{\mathcal{P}}_{\psi}^{*}$. Using the KT equations (36a) and (36b),

$$
\dot{\mathcal{P}}_{\psi}^{*}=\left(\dot{p}_{\mathrm{a}}^{*}-\frac{1}{2} S_{\mathrm{bc}}^{*} R_{\mathrm{da}}^{\mathrm{bc}} \dot{\gamma}^{\mathrm{d}}\right) A^{\mathrm{a}}+\frac{1}{2}\left(\dot{S}_{\mathrm{ab}}^{*}+2 \dot{\gamma}_{\mathrm{a}} p_{\mathrm{b}}^{*}\right) B^{\mathrm{ab}} .
$$

This is closely analogous to (37). The left-hand side is parameterized entirely by $A_{a}$ and $B_{\mathrm{ab}}$, so varying these quantities determines all of the corrections to the Papapetrou equations.

Two definitions have now been suggested for the momenta of an extended body. The first - summarized by (24) and (35) - is closely related to the one given by Dixon 25, 28, 29. It is well-adapted to the construction of multipole moments for $T^{a b}$ that intrinsically take into account stress-energy conservation. Mass centers defined from these momenta are known to have most of the properties one might expect [12, 35. The boundary of the worldtube $W$ isn't important as long as it lies outside of the matter distribution under discussion. There is no vacuum momentum under this definition. Unfortunately, there does not appear to be any exact analog of Gauss' law either. The momenta of a matter distribution (and changes to it) must be computed by integrating over 3-volumes. The generalized Komar integrals defined by (72) have complementary characteristics. Their main advantage is in having a direct interpretation analogous to Gauss' law. Changes in the component of an isolated body's momentum generated by $\psi^{a}$ only depends on the gravitational flux $j_{\psi}^{a}$ passing through the surface $\partial W$. The mass and angular momenta expected from this definition also agree with commonlyaccepted notions at least in appropriately symmetric spacetimes. It is potentially problematic that $\mathcal{P}_{\psi}^{*}$ includes what is effectively a vacuum energy. These scalars usually depend on the spatial extent of $W$ even when its boundary lies far outside of any matter distribution. This can make it difficult to neatly separate the properties of disjoint matter distributions, although similar situations are found even in ordinary electromagnetism. It might be conceptually simpler to extend $\partial W$ to infinity, although it is unlikely that all of the bitensors used here would remain well-defined. There might also be convergence problems. Related concepts presented in [36] could be more useful for defining momenta over very large regions.

Some insight into the behavior of the $\mathcal{P}_{\psi}^{*}$ defined here can be gained by computing it for very small spheres. To be specific, let $C(r, s)$ be the closed 2-surface on $\Sigma(s)$ satisfying $X^{\mathrm{a}} X_{\mathrm{a}}=r^{2}$ for some $r>0$. This is effectively a sphere of proper radius $r$ centered at the point $\gamma(s)$. It follows from (38) and (70) that $j_{\psi}^{a}$ is negligible for small radii in the presence of matter. The momentum inside $C$ is approximately

$$
P_{\xi}^{*} \simeq \frac{8 \pi}{3} r^{3}\left(T^{\mathrm{a}}{ }_{\mathrm{b}} A^{\mathrm{b}}-\frac{1}{2} A^{\mathrm{a}} T^{\mathrm{b}} \mathrm{b}\right) \nabla_{\mathrm{a}} \tau+\mathrm{O}\left(r^{4}\right) .
$$

It is perhaps more interesting to consider regions that are locally devoid of matter. These can be understood from the behavior of the gravitational current. Its approximate behavior near $\Gamma$ is easily calculated from (33) and (70). To lowest nontrivial order,

$$
j_{\psi}^{a} \simeq-\frac{1}{8 \pi} \sigma^{a}{ }_{\mathrm{a}} X^{\mathrm{b}}\left[\mathcal{L}_{\psi} R^{\mathrm{a}}{ }_{\mathrm{b}}+\frac{1}{3}\left(g^{\mathrm{ac}} R^{\mathrm{d}}{ }_{\mathrm{b}}+2 R_{\mathrm{b}}{ }^{\mathrm{cad}}\right) \mathcal{L}_{\psi} g_{\mathrm{cd}}\right]+\mathrm{O}\left(X^{2}\right) .
$$


This vanishes in vacuum for Killing-type Jacobi fields. A little more calculation finds the same conclusion at order $X^{2}$ as well. This contrasts sharply with other quasilocal notions of vacuum momentum in general relativity. An extensive review of these concepts may be found in 37. As remarked there, the energy contained in small spheres has been calculated using several different definitions. The generic result is that it is proportional to the Bel-Robinson tensor, and scales like $r^{5}$. If this were true for the definition suggested here, terms quadratic in the curvature would appear at order $X^{2}$ in the current. These are not found. Vacuum momenta should really only be associated with Killing-type symmetries, so the relevant currents defined here decrease at least as fast as $r^{6}$ as $r \rightarrow 0$. Other definitions in the literature find more energy in very small spheres. It is not clear how to interpret this, although it might have interesting consequences for the use of near zones and related concepts connected to the mechanics of compact bodies.

\section{An example: gravitational plane waves}

The discussion so far has mainly focused on the behavior of generalized symmetries near the point or worldline used to construct them. With the exception of general identities like (A.7), it has not been clear what happens to these vector fields far away from their origins. It is therefore useful to consider an example. Given (17), the Jacobi fields can all be calculated simply by differentiating the world function. This makes it convenient to consider spacetimes where $\sigma$ is known exactly. Essentially the only examples of this type are $p p$-waves or assorted cosmological models (see [38] and references cited therein).

In the interest of understanding the generalized Komar momenta (72) in a vacuum spacetime, only $p p$-waves will be considered here. Coordinates may be introduced such that the metric satisfies

$$
\mathrm{d} s^{2}=-2 \mathrm{~d} u \mathrm{~d} v+a(u) \mathrm{d} x^{2}+b(u) \mathrm{d} y^{2} .
$$

It can then be shown that one-half of the geodesic distance between points with coordinates $(u, v, x, y)$ and $(\mathrm{u}, \mathrm{v}, \mathrm{x}, \mathrm{y})$ is given by [39, 40.

$$
\sigma=\frac{1}{2}\left[\alpha(u, \mathrm{u})(x-\mathbf{x})^{2}+\beta(u, \mathrm{u})(y-\mathrm{y})^{2}\right]-(u-\mathrm{u})(v-\mathrm{v}),
$$

where

$$
\alpha(u, \mathbf{u})=\frac{u-\mathbf{u}}{\int_{\mathbf{u}}^{u} a^{-1}(w) \mathrm{d} w} ; \quad \beta(u, \mathbf{u})=\frac{u-\mathbf{u}}{\int_{\mathbf{u}}^{u} b^{-1}(w) \mathrm{d} w} .
$$

It is clear by inspection that $\partial / \partial x, \partial / \partial y$, and $\partial / \partial v$ are exact Killing vectors. They are not the only ones. All nontrivial spacetimes in this class admit between five and seven linearly-independent Killing fields.

Most $p p$-waves are effectively "null dust" solutions of Einstein's equation, although there are vacuum examples as well. One of these is given by

$$
a(u)=\cos ^{2}(\lambda u) ; \quad b(u)=\cosh ^{2}(\lambda u)
$$

where we assume that $|\lambda u|<\pi / 2$ in order to avoid the two coordinate singularities. This represents a simple plane-fronted gravitational wave with amplitude $\lambda$. The only non-vanishing components of the curvature are

$$
C_{u x u}^{x}=C_{u y u}^{y}=\lambda^{2} .
$$


It is trivial to modify this spacetime to be flat for (say) $u<0$ [41, although impulsive waves of this type will not be discussed here.

Given (79), it is straightforward to explicitly compute the Jacobi propagators $H^{a}{ }_{\mathrm{b}} \sigma^{\mathrm{b}}{ }_{\mathrm{a}}$ and $H^{a}{ }_{\mathrm{a}} \sigma_{\mathrm{b}}$ using (14). This can be done for any choice of $a(u)$ and $b(u)$, although we will specialize to the case defined by (81). The results are not particularly enlightening to write down in detail, although they have some interesting consequences. First, all Jacobi fields are found to be exactly Killing if their first derivatives vanish at the base point (denoted as usual with sans-serif font). The Jacobi fields $H^{a}{ }_{[x} \sigma_{\mathrm{v}]}$ and $H^{a}{ }_{[\mathrm{y}} \sigma_{\mathrm{v}]}$ are also Killing. This identifies six independent Killing fields. It also implies that the linear gravitational momentum $p_{\mathrm{a}}^{*}$ defined in (74) must vanish. This can be taken to imply (unsurprisingly) that the gravitational wave has zero rest mass: $\left|p^{*}\right|^{2}=0$.

There remain four non-Killing Jacobi fields with skew-symmetric $B_{\mathrm{ab}}$. The one associated with spatial rotations in the $x-y$ plane is relatively simple to write down when $\mathrm{u}=0$ :

$$
\begin{array}{r}
\psi_{\mathrm{xy}}^{a}=2 H_{[\mathrm{x}}^{a} \sigma_{\mathrm{y}]}=-(y-\mathrm{y})\left(\frac{\tan (\lambda u)}{\tanh (\lambda u)}\right) \frac{\partial}{\partial x}+(x-\mathrm{x})\left(\frac{\tanh (\lambda u)}{\tan (\lambda u)}\right) \frac{\partial}{\partial y} \\
+\lambda(x-\mathrm{x})(y-\mathrm{y})\left(\frac{\tanh (\lambda u)-\tan (\lambda u)}{\tan (\lambda u) \tanh (\lambda u)}\right) \frac{\partial}{\partial v} .
\end{array}
$$

This clearly reduces to its expected form when $u \rightarrow \mathrm{u}$. The degree to which it succeeds in being a genuine Killing field may be estimated by noting that

$$
\left|\mathcal{L}_{\psi_{x y}} g_{a b}\right|^{2}=\left(\frac{\cos (2 \lambda u)+\cosh (2 \lambda u)-2}{\sqrt{2} \sin (\lambda u) \sinh (\lambda u)}\right)^{2} \simeq \frac{8}{9}(\lambda u)^{4}+\mathrm{O}\left(u^{8}\right)
$$

when $u=0$. The quadratic growth estimate here is typically quite good even near the coordinate singularities. There is little qualitative change in the nature of this expression if $u \neq 0$. More interestingly, the gravitational current (70) associated with $\psi_{\mathrm{xy}}^{a}$ always vanishes. This suggests that an observer would not be compelled to ascribe any $x y$ component of angular momentum to gravitational waves with the given form. $H^{a}{ }_{[\mathrm{u}} \sigma_{\mathrm{v}]}$ has similar properties. It satisfies an equation almost identical to (84), and the gravitational current generated by it always vanishes.

More interesting are the remaining two Killing-type Jacobi fields $\psi_{\mathrm{xu}}^{a}=2{H^{a}}_{[\mathrm{x}} \sigma_{\mathrm{u}]}$ and $\psi_{\mathrm{yu}}^{a}=2 H_{[\mathrm{y}}^{a} \sigma_{\mathrm{u}]}$. Specializing again to the case $\mathbf{u}=0$,

$$
\left|\mathcal{L}_{\psi_{\mathrm{xu}}} g_{a b}\right|^{2} \simeq\left|\mathcal{L}_{\psi_{\mathrm{yu}}} g_{a b}\right|^{2} \simeq \frac{2}{3} \lambda^{4} u^{2}\left(\left[(x-\mathrm{x})^{2}+(y-\mathrm{y})^{2}\right]+\frac{2}{3}(v-\mathrm{v}) u\right)+\mathrm{O}\left(u^{4}\right) .
$$

Unlike the expansion in (84), this approximation fails long before $|\lambda u| \rightarrow \pi / 2$. In general, the two magnitudes on the left-hand side have distinct behaviors that strongly depend on u. Oscillations generically arise as $u$ is varied, for example. See Fig. 1.

There are gravitational currents associated with both $\psi_{\mathrm{xu}}^{a}$ and $\psi_{\mathrm{yu}}^{a}$. Applying (70) and expanding near $\mathrm{u}=0$,

$$
j_{\psi_{\mathrm{xu}}}^{a}=\frac{\lambda^{6}}{180 \pi} u^{4}\left[u \frac{\partial}{\partial x}+(x-\mathrm{x}) \frac{\partial}{\partial v}\right]+\mathrm{O}\left(u^{6}\right)
$$

Similarly,

$$
j_{\psi_{\text {yu }}}^{a}=-\frac{\lambda^{6}}{180 \pi} u^{4}\left[u \frac{\partial}{\partial y}+(y-\mathrm{y}) \frac{\partial}{\partial v}\right]+\mathrm{O}\left(u^{6}\right)
$$




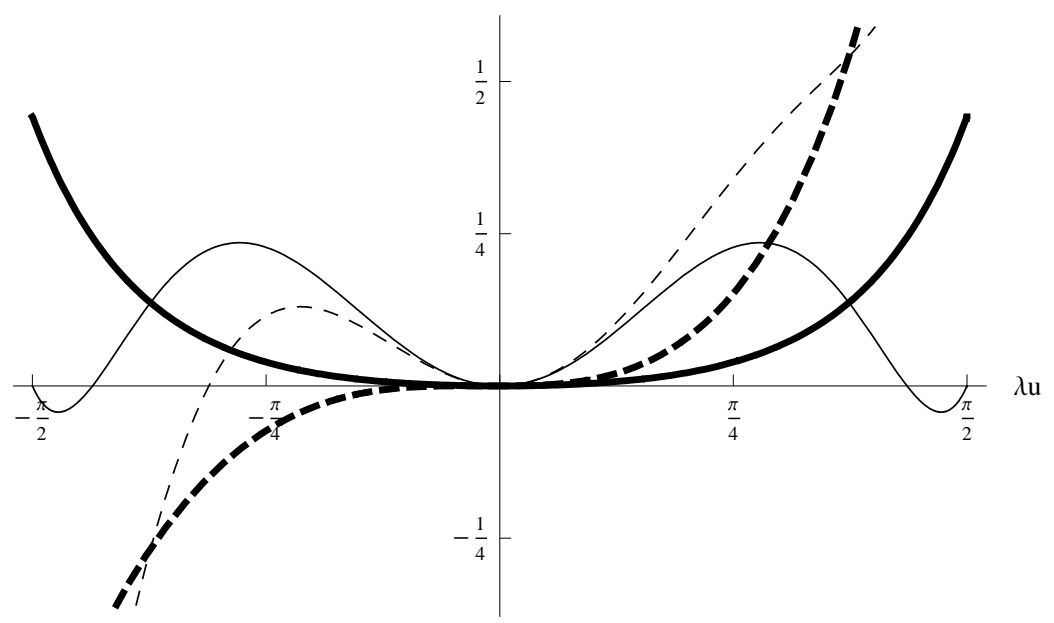

Figure 1. Plots of $\left|\mathcal{L}_{\psi_{\mathrm{xu}}} g_{a b}\right|^{2}$ for a gravitational plane wave described by (78) and (81). The origin is assumed to be at $u=0$. Both solid curves assume that $v-v=0$. The dashed ones use $\lambda(v-v)=1 / 2$ instead. Both thicker curves set $x-\mathrm{x}=0$ and $\lambda(y-\mathrm{y})=1 / 4$. The thinner ones use $\lambda(x-\mathrm{x})=1$ and $y-\mathrm{y}=0$. Plots for $\left|\mathcal{L}_{\psi_{\mathrm{yu}}} g_{a b}\right|^{2}$ look very similar unless $\mathrm{u} \neq 0$.

These expansions are qualitatively accurate throughout the region of interest. It is now clear from (73) and (74) that the only non-vanishing gravitational momenta (associated with Killing-type GACs) are

$$
S_{\text {хu }}^{*}=\int_{\Sigma} j_{\psi_{x u}}^{a} \mathrm{~d} S_{a} ; \quad S_{\mathrm{yu}}^{*}=\int_{\Sigma} j_{\psi_{y u}}^{a} \mathrm{~d} S_{a} .
$$

The rates at which these quantities change depends on the relevant fluxes through $\partial \Sigma$. Regardless, the magnitude of the angular momentum tensor always vanishes. Intuitively, these statements might be taken to mean that the gravitational wave has a "mass dipole moment" equal to its one non-vanishing component of ordinary angular momentum.

The results here could be straightforwardly extended to much more general $p p$ wave (and other) spacetimes. The most interesting point is perhaps the calculation of explicit gravitational currents in a vacuum spacetime. In these cases, the general results obtained in Sect. 4.2 only state that $j^{a}$ will decrease no slower than $X^{3}$ as $X \rightarrow 0$. The example here scales like $X^{5}$. Although this conclusion would probably not be preserved in more complicated spacetimes, it shows that momenta not arising from stress-energy tensors can sometimes be ignored in remarkably large regions.

\section{Conclusions}

Two different notions of approximate affine collineations have been introduced. One has the physical interpretation of capturing symmetry principles in a normal neighborhood of a point, while the other is adapted to the measurements of a particular observer. Flows generated by both of these objects leave $\sigma^{\mathrm{a}}(x, \gamma)$ invariant. This has the simple interpretation that Jacobi fields preserve Riemann normal coordinates. GACs do the same for the spatial components of Fermi normal coordinate systems. 
These objects always exist, and each forms a 20-dimensional group. Individual elements may be interpreted using the values of the field and its first derivatives at the appropriate base point. The only caveat to this is that a GAC which might initially appear to be purely translational could slowly acquire some rotational and boost-type components. This mixing is essential in order to ensure that the fields nearly satisfy (3) near the observer's worldline.

The relevance of these definitions ultimately lies in their applications. The approximate symmetries introduced here have been used to write down analogs of the typical conservation laws applying to geodesics in spacetimes admitting affine collineations. Some of the resulting parameters are exact constants of motion, while others are only expected to vary slowly near the preferred point or observer. Regardless, they may be used to classify and derive geodesics in certain regions. Similar results have also been discussed in connection with extended matter distributions. This led to natural notions for the linear and angular momenta of a spacetime volume as viewed in a particular frame. There seems to be some disagreement with other quasilocal notions of gravitational momenta, so it is not clear how the definition here should be interpreted. It is unknown if it has any positivity or related characteristics.

Concepts discussed here might also be applied to simplify perturbation theory off of some background geometry possessing an exact affine collineation. It could be useful, for example, to uniquely construct GACs with respect to a center-of-mass worldline that coincides with exact timelike or axial Killing fields in the unperturbed geometry. Center-of-mass trajectories might also be estimated using notions of approximate stationarity. More concretely, an analysis of the quantities $\mathcal{P}_{\xi}$ defined in (35) can be shown to provide significant insights into the effects of self-forces and self-torques on isolated bodies. Details are presented elsewhere [42.

\section{Acknowledgments}

I am grateful for many helpful discussions and comments from Robert Wald and Samuel Gralla. This work was supported by NSF grant PHY04-56619 to the University of Chicago.

\section{Appendix: Properties of Jacobi fields}

The Jacobi fields and generalized affine collineations satisfy a number of useful identities that both simplify various calculations and further motivate their identification as generators of approximate symmetries. The most basic of these results is probably (18) or its analog (38). Symmetries defined with respect to a given point are exactly affine at that point. Symmetries defined with respect to a given worldline are exactly affine on that worldline. Nothing is said about the behavior of these fields away from these regions. It would be much more interesting if constraints could be placed on $\nabla_{a} \mathcal{L}_{\psi} g_{b c}$ throughout the volume $N$ where it is defined. This is indeed possible. Jacobi fields generate diffeomorphisms that exactly preserve certain geometric objects in all locally well-behaved spacetimes. This fact can be used to derive some properties

of $\mathcal{L}_{\psi} g_{a b}$ and its derivatives even when $x \neq \gamma$. Somewhat weaker comments can also be made regarding each GAC $\xi^{a}$. Beyond this, approximations for these vector fields can be generically obtained near their origins. A few results of this type have been 
derived before for Killing-type Jacobi fields [28, 29]. These will be generalized here while also introducing a number of apparently new results.

The fundamental starting point is the observation that $\sigma^{\mathrm{a}}(x, \gamma)$ must remain unchanged if both of its arguments are perturbed with a Jacobi field defined about $\gamma$ 29]. More concisely,

$$
\mathcal{L}_{\psi} \sigma^{\mathrm{a}}(x, \gamma)=\psi^{a} \sigma^{\mathrm{a}}{ }_{a}+A^{\mathrm{b}} \sigma^{\mathrm{a}}{ }_{\mathrm{b}}-\sigma^{\mathrm{b}} B_{\mathrm{b}}{ }^{\mathrm{a}}=0 .
$$

This means that Riemann normal coordinate grids are left invariant under infinitesimal transformations generated by $\psi^{a}$. It can be verified using (14) and (19). The solutions to (A.1) are seen to all have the form (17) derived for the Jacobi fields. The undifferentiated world function is left unchanged only under the action of Killingtype Jacobi fields. More generally,

$$
\mathcal{L}_{\psi} \sigma(x, \gamma)=\sigma^{\mathrm{a}} \sigma^{\mathrm{b}} B_{(\mathrm{ab})} .
$$

In the special case of a generalized homothetic vector field satisfying (28), this reduces to the particularly simple form

$$
\mathcal{L}_{\psi_{\mathrm{H}}} \sigma=2 c \sigma .
$$

Returning to the general case, several additional identities may be derived from (A.1) and (A.2) by differentiation. It is clear that a single covariant derivative with respect to $x$ will commute with the Lie derivatives in both of these equations. Using this fact shows that

$$
\mathcal{L}_{\psi} \sigma^{\mathrm{a}}{ }_{a}=\mathcal{L}_{\psi} H^{a}{ }_{\mathrm{a}}=0,
$$

and

$$
\mathcal{L}_{\psi} \sigma_{a}=2 \sigma^{\mathrm{a}} \sigma^{\mathrm{b}}{ }_{a} B_{(\mathrm{ab})} .
$$

It then follows from (27) and (A.4) that

$$
\mathcal{L}_{\psi} \sigma^{a}=0 .
$$

Comparing this to what would be expected from (A.5) produces the very useful identity

$$
\sigma^{a} \mathcal{L}_{\psi} g_{a b}=2 \sigma^{\mathrm{a}} \sigma^{\mathrm{b}}{ }_{b} B_{(\mathrm{ab})} .
$$

This result is central to the interpretation of Jacobi fields as generalized symmetry generators. It applies most clearly to Killing-type vectors $\psi_{\mathrm{K}}^{a}$. The right-hand side then vanishes, providing a strong restriction on how much these fields can fail to satisfy Killing's equation away from $\gamma$.

More generally, there should exist an analog of (A.7) that constrains $\nabla_{a} \mathcal{L}_{\psi} g_{b c}$ for all Jacobi fields. Expressions of this form are easily obtained by directly differentiating (A.7). Applying identities like (27) to the result then shows that

$$
\sigma^{a} \sigma^{b}\left[2 \nabla_{(a} \mathcal{L}_{\psi} g_{b) c}-\nabla_{c} \mathcal{L}_{\psi} g_{a b}\right]=0
$$

and

$$
\sigma^{a} \sigma^{c} \nabla_{a} \mathcal{L}_{\psi} g_{b c}=2 \sigma^{\mathrm{a}}\left(\sigma^{\mathrm{b}}{ }_{b}-\sigma^{\mathrm{b}}{ }_{c} \sigma^{c}{ }_{b}\right) B_{(\mathrm{ab})} .
$$

The first of these equations ensures that that there is always some sense in which every Jacobi field is approximately affine. It is actually identical to (21). One trivial consequence is that

$$
\sigma^{a} \sigma^{b} \sigma^{c} \nabla_{a} \mathcal{L}_{\psi} g_{b c}=0 .
$$


It means that the "purely radial" component of (3) holds everywhere that $\psi^{a}$ is defined. Similar statements can also be made using (A.9). The right-hand side of that equation vanishes for Killing or homothetic-type Jacobi fields, although interesting remarks can be made even in the general case. Temporarily consider points that are spacelikeseparated from $\gamma$, so $\sigma>0$. It is then clear that in normal coordinates, the appropriate components of $\nabla_{a} \mathcal{L}_{\psi} g_{b c}$ fall off roughly like $1 / \sqrt{\sigma}$ far away from the origin. These quantities also have a simple scaling behavior near $\gamma$. Given that all third derivatives of the world function vanish when $x \rightarrow \gamma$, the term

$$
\sigma^{\mathrm{b}}{ }_{b}-\sigma^{\mathrm{b}}{ }_{c} \sigma^{c}{ }_{b}=\sigma^{a} \sigma^{\mathrm{b}}{ }_{a b}
$$

in (A.9) must go to zero at least as fast as $\sigma$ in this limit. It then follows that the portions of $\nabla_{a} \mathcal{L}_{\psi} g_{b c}$ constrained in that equation should grow no faster than $\sqrt{\sigma}$ for $x$ very near $\gamma$.

A more explicit connection can actually be made between $\nabla_{a} \mathcal{L}_{\psi} g_{b c}$ and derivatives of the world function. Several remarks have already been made about the interpretation of various results here in terms of normal coordinate systems. Since (3i) is essentially the condition that leaves the Levi-Civita connection invariant, one might expect an appropriate result to arise from studying the Christoffel symbols in normal coordinates. These are related to the bitensor $\sigma^{\mathrm{a}}{ }_{a b}$. Lie derivatives of this quantity can be computed by directly differentiating (A.4). Simplifying the result requires the relation

$$
\nabla_{b} \nabla_{a} \psi_{c}=R_{c a b d} \psi^{d}+\frac{1}{2}\left[2 \nabla_{(a} \mathcal{L}_{\psi} g_{b) c}-\nabla_{c} \mathcal{L}_{\psi} g_{a b}\right] .
$$

This is obtained by repeatedly applying Ricci's identity, and actually applies for any vector field. It is a generalization of (22) and the well-known expression for second derivatives of a Killing field. Combining (14), (A.4), and (A.12) shows that

$$
\nabla_{a} \mathcal{L}_{\psi} g_{b c}=2 H_{(c|\mathrm{a}|} \mathcal{L}_{\psi} \sigma^{\mathrm{a}}{ }_{b) a} .
$$

This is essentially the expected result.

There is at least one more quantity derived from the world function that has a simple interpretation in normal coordinates. That is the van Vleck determinant

$$
\Delta(x, \gamma)=\frac{\operatorname{det}\left(-\sigma_{\mathrm{a} a}\right)}{\sqrt{-g(x)} \sqrt{-g(\gamma)}} .
$$

This two-point scalar arises as part of the volume element in these charts. It also appears in connection with the focusing of geodesic congruences and in Green functions associated with common wave equations [24. It is clear from the definition (A.14) that

$$
\nabla_{b} \ln \Delta=-H^{a}{ }_{a} \sigma^{\mathrm{a}}{ }_{a b} .
$$

Derivatives with respect to $\gamma$ are identical if the index $b$ in this equation is changed to a b. Simplifying with (A.4) then shows that

$$
\mathcal{L}_{\psi} \ln \Delta=\nabla_{\mathrm{a}} \psi^{\mathrm{a}}-\nabla_{a} \psi^{a}=B^{\mathrm{a}}{ }_{\mathrm{a}}-\frac{1}{2} g^{a b} \mathcal{L}_{\psi} g_{a b} .
$$

The results obtained so far apply everywhere that the generalized symmetries are defined. It is sometimes more useful to derive explicit approximations for the Jacobi fields. These can be found in various ways. Perhaps the most obvious procedure is to evaluate (17) in a normal coordinate system with origin $\gamma$. The second term involving $B^{\text {ba }}$ then reduces to a linear combination of the coordinate functions. This is exact. 
The factor of $\sigma^{\mathrm{a}} \mathrm{b}$ appearing in the other term does not simplify in these coordinates. It reduces to the identity at the origin, although its behavior further away must be computed in some other way. For $X^{\text {a }}$ very small, known Taylor expansions for $\sigma^{\mathrm{a}}{ }_{\mathrm{b}}$ could be used 24]. There are also integral approximations neglecting all terms nonlinear in the curvature [23, 30. Either of these methods can be used to find explicit expressions for $\psi^{a}$ or $\xi^{a}$ in appropriate situations, although it is often more interesting to directly compute Lie derivatives of the metric with respect to these vector fields. The viewpoint here will be to derive a Taylor series for $\mathcal{L}_{\psi} g_{a b}$ centered at $\gamma$. This expansion will be in powers of the radial vector $-\sigma^{a}$. The first two terms are clear from (19) and (38). Going to higher orders requires knowledge of $\left[\nabla_{a} \nabla_{b} \mathcal{L}_{\psi} g_{c d}\right]$, $\left[\nabla_{a} \nabla_{b} \nabla_{c} \mathcal{L}_{\psi} g_{d f}\right]$, and so on. The bracket notation here was defined in (41). In any case, limits like these may be computed from (A.13). Results such as (A.12) can further be used to derive the full third or fourth covariant derivatives of $\psi^{a}$ in the coincidence limit.

First consider third derivatives. From (A.12), $\nabla_{a} \nabla_{b} \mathcal{L}_{\psi} g_{c d}$ involves $\left[\nabla_{b} H^{a}{ }_{a}\right]$ and $\left[\nabla_{d} \mathcal{L}_{\psi} \sigma^{\mathrm{a}}{ }_{b c}\right]$. All third derivatives of the world function vanish at coincidence, so only the second term is important here. Some manipulation shows that

$$
\left[\nabla_{d} \mathcal{L}_{\psi} \sigma^{\mathrm{a}}{ }_{b c}\right]=\mathcal{L}_{\psi}\left[\sigma^{\mathrm{a}}{ }_{b c d}\right] .
$$

The quantity being differentiated on the right-hand side is known to have the form 24, 23, 30.

so

$$
\left[\sigma_{b c d}^{\mathrm{a}}\right]=-\frac{2}{3} R_{(b c) d}^{\mathrm{a}},
$$

$$
\left[\nabla_{a} \nabla_{b} \mathcal{L}_{\psi} g_{c d}\right]=\frac{2}{3}\left(R_{\mathrm{ab}(\mathrm{c}}{ }^{\mathrm{f}} \mathcal{L}_{\psi} g_{\mathrm{d}) \mathrm{f}}+g_{\mathrm{f}(\mathrm{c}} \mathcal{L}_{\psi} R_{\mathrm{d}) \mathrm{ab}}{ }^{\mathrm{f}}\right) .
$$

Lie derivatives here have their usual expansions in terms of $A_{\mathrm{a}}, B_{\mathrm{ab}}, R_{\mathrm{abc}}{ }^{\mathrm{d}}$, and $\nabla_{\mathrm{f}} R_{\mathrm{abc}}{ }^{\mathrm{d}}$. The first term clearly vanishes for Killing-type Jacobi fields. The other depends on how close $\psi^{a}$ comes to being a curvature collineation (satisfying $\left.\mathcal{L}_{Y_{\mathrm{C}}} R_{a b c}{ }^{d}=0\right)$ at $\gamma$.

It is not too difficult to compute one more derivative of $\mathcal{L}_{\psi} g_{a b}$. This is again derived from $(\underline{A .13})$. Noting that

$$
\left[\mathcal{L}_{\psi} \sigma^{\mathrm{a}}{ }_{b c}\right]=\mathcal{L}_{\psi}\left[\sigma^{\mathrm{a}}{ }_{b c}\right]=0,
$$

one finds

$$
\left[\nabla_{a} \nabla_{b} \nabla_{c} \mathcal{L}_{\psi} g_{d f}\right]=2\left[\nabla_{a} \nabla_{b} \mathcal{L}_{\psi} \sigma^{\mathrm{h}}{ }_{c(d}\right] g_{\mathrm{f}) \mathrm{h} .}
$$

The two covariant derivatives here may be commuted inside the Lie derivative. A number of remainder terms arise, although these all vanish on account of (A.12) and (A.18). This leaves an expression dependent only on $\left[\sigma_{b c d f}^{\mathrm{a}}\right]$. As noted in [23,

$$
\left[\sigma_{a b c d f}\right]=\nabla_{(\mathrm{f}} R_{\mathrm{d})(\mathrm{ab}) \mathrm{c}}+\frac{1}{2} \nabla_{\mathrm{c}} R_{\mathrm{d}(\mathrm{ab}) \mathrm{f}} .
$$

A straightforward application of Synge's rule together with (A.18) shows that

$$
\left[\sigma_{b c d f}^{\mathrm{a}}\right]=\frac{1}{6} \nabla^{\mathrm{a}} R_{\mathrm{b}(\mathrm{df}) \mathrm{c}}-\nabla_{(\mathrm{f}} R_{\mathrm{d})(\mathrm{bc})}{ }^{\mathrm{a}} .
$$

Combining all of these results,

$$
\begin{aligned}
{\left[\nabla_{a} \nabla_{b} \nabla_{c} \mathcal{L}_{\psi} g_{d f}\right]=} & \frac{1}{3} g_{\mathrm{h}(\mathrm{d}} \mathcal{L}_{\psi} \nabla^{\mathrm{h}} R_{\mathrm{f})(\mathrm{ab}) \mathrm{c}}-g_{\mathrm{hd}} \mathcal{L}_{\psi} \nabla_{(\mathrm{a}} R_{\mathrm{b})(\mathrm{cf})}{ }^{\mathrm{h}} \\
& -g_{\mathrm{hf}} \mathcal{L}_{\psi} \nabla_{(\mathrm{a}} R_{\mathrm{b})(\mathrm{cd})}{ }^{\mathrm{h}} .
\end{aligned}
$$

Taylor expansions derived from these limits are given by (32), (33), (52), and (53). 


\section{References}

[1] Matzner R A 1968 J. Math. Phys. 91657

[2] Bona C, Carot J and Palenzuela-Luque C 2005 Phys. Rev. D 72124010

[3] Yano K and Bochner S 1953 Curvature and Betti Numbers (Princeton, NJ: Princeton University Press)

[4] Komar A 1962 Phys. Rev. 1271411

[5] Komar A 1963 Phys. Rev. 1291873

[6] York J W 1974 Ann. Inst. Henri Poincaré 21319

[7] Caudill M, Cook G B, Grigsby J D, and Pfeiffer H P 2006, Phys. Rev. D 74064011

[8] Dreyer O, Krishnan B, Shoemaker D, and Schnetter E 2003, Phys. Rev. D 67, 024018

[9] Cook G B and Whiting B F 2007 Phys. Rev. D 76 041501(R)

[10] Dain S 2004 Phys. Rev. Lett. 93231101

[11] Wald R M 1984 General Relativity (Chicago, IL: University of Chicago Press)

[12] Schattner R and Streubel M 1981 Ann. Inst. Henri Poincaré 34117

[13] Zafiris E 1997 J. Math. Phys. 385854

[14] Stephani H, Kramer D, MacCallum M, Hoenselaers C and Herlt E 2002 Exact Solutions of Einstein's Field Equations (Cambridge: Cambridge University Press)

[15] Hall G S 2004 Symmetries and Curvature Structure in General Relativity (Singapore: World Scientific)

[16] Carr B J and Coley A A 1999 Class. Quantum Grav. 16 R31

[17] Hall G S 1988 Gen. Rel. Grav. 20399

[18] Garfinkle D 1987 J. Math. Phys. 2828

[19] Hojman S, Nuñez L, Patiño A, and Rago H 1986 J. Math. Phys. 27281

[20] Katzin G H, Levine J and Davis W R 1969 J. Math. Phys. 10617

[21] Collinson C D 1970 Gen. Rel. Grav. 1137

[22] Ashtekar A and Magnon A 1984 Class. Quantum Grav. 1 L39

[23] Synge J L 1966 Relativity: the general theory (Amsterdam: North-Holland)

[24] Poisson E 2004 Living Rev. Rel. 76

[25] Dixon W G 1970 Proc. R. Soc. A 314499

[26] Caviglia G, Zordan C and Salmistraro F 1982,

[27] Caviglia G, Salmistraro F and Zordan C 1982 J. Math. Phys. 232346

[28] Dixon W G 1974 Phil. Trans. R. Soc. A 27759

[29] Dixon W G 1979 Extended bodies in general relativity; their description and motion Isolated Gravitating Systems in General Relativity ed. J Ehlers (Amsterdam: North-Holland)

[30] de Felice F and Clarke C J S 1992 Relativity on Curved Manifolds (Cambridge: Cambridge University Press)

[31] Ehlers J and Rudolph E 1977 Gen. Rel. Grav. 8197

[32] Patino A 1993 ApSS 20877

[33] Katzin G H and Levine J 1981 J. Math. Phys. 221878

[34] Streubel M and Schattner R 1981, Ann. Inst. Henri Poincaré 34145

[35] Schattner R 1979 Gen. Rel. Grav. 10 377; 10395

[36] Streubel M and Schattner R 1979 Connection between asymptotic structure and material sources Proceedings of the 3rd Gregynog Workshop on Gravitational Radiation Theory ed M Walker (Munich: Max Planck Institut für Physik und Astrophysik)

[37] Szabados L B 2004, Living Rev. Rel. 74

[38] Roberts M D 1993 Astro. Lett. and Communications 28349

[39] Günther P 1965 Arch. Rat. Mech. and Analysis 18103

[40] Friedlander F G 1975 The Wave Equation on a Curved Space-Time (Cambridge: Cambridge University Press)

[41] Griffiths J B 1991 Colliding Plane Waves in General Relativity (Oxford: Oxford University Press)

[42] Harte A I 2008 Preprint arXiv:0807.1150 